\title{
Autonomous pressure control in water distribution networks
}

\author{
B. Plemper ${ }^{1} \&$ M. Redman ${ }^{2}$ \\ ${ }^{I}$ Northumbrian Water, UK \\ ${ }^{2}$ Flow Control, Industrial Valves Limited, UK
}

\begin{abstract}
The configuration of most water distribution systems have evolved over time developing almost organically as additional population centres have expanded the network. Unless periodical reviews of the distribution infrastructure are carried out water networks become chaotic resulting in inefficiencies in both water supply transfer capacity and distribution costs.

Maintenance is an ongoing process for all UK water companies and at present most companies are compiling programs to replace aging key assets within their distribution systems. It would be prudent under these circumstances to consider if the configuration of the distribution network as a whole can be improved when asset replacement is scheduled.

At Northumbrian Water, novel control valve design and new control paradigms have been combined to develop a self-controlling water distribution system that simplifies network control improves pressure management and reduces distribution costs, leakage and burst rates.

Keywords: water distribution networks, asset lifetime, automation, cost minimisation, valve technology, pressure management.
\end{abstract}

\section{Introduction}

As assets within water distribution networks near the end of their lifetime a formulated approach is required, to produce a replacement plan that will create an integrated hydraulic network. The plan set out below has been developed to allow water companies to achieve this goal and attain an automated water distribution system which will deliver water at a minimum cost. 
The strategy considers each network component as it approaches the end of its asset life and designs the succeeding asset to fulfil the requirements of local environment and also those of the system as a whole.

Recent developments in valve technology have allowed new pressure management techniques to be create that can automate network control and substantially reduce burst frequencies and levels of leakage. Trials of these techniques carried out at Northumbrian Water in conjunction with IVL Flow Control, have produced a robust network control philosophy, which attains efficient network management in conjunction with minimised levels of leakage and burst rate.

This aim can be achieved by creating, a network of intelligent control valves situated at critical nodes throughout the distribution system. From this, a calmed pressure system will ensue. This integrated system will minimise both "no water" (DG3) and low pressure (DG2) incidents, extended asset lifetime and reduce maintenance costs. Additionally the system will regulate flow and pressure, reduce both leakage and burst rate and be self-optimising on a cost and water quality basis.

High levels of leakage and burst frequency in potable water distribution systems are costly in both economic and environmental terms [2]. Any process that can minimise this wastage is seen as a positive practice to employ by both water companies and regulatory bodies alike [3].

\section{Methodology and model}

This section deals with the rational and concepts involved in the development of an integrated autonomous potable water distribution system. The control theory in a system such as this is centred on the concept of self-optimising nodes. These nodes are key points within a hydraulic network through which the flow of water is controlled. A self-optimising node reacts to the demand variations within the network and modulates the flow of water through the system by regulating the pressure on both sides of the valve.

The model devised here is, as far as can be ascertained, original and is not used by other water companies as an automated control solution.

\subsection{Demand control valve (DCV)}

The demand control valve is a modified flow control valve [4]. At each selfoptimising node a DCV controls the flow of water through the node (see Figure 1). The DCV operates autonomously via two hydraulic pilots [5].

The inlet pilot is dominant and operates as a pressure sustaining valve (PSV). It will only operate if the upstream pressure is above a set value.

The outlet pilot is secondary and operates as a pressure reducing valve (PRV). The PRV can only operate if the upstream PSV is active and the downstream pressure falls below a set value. 


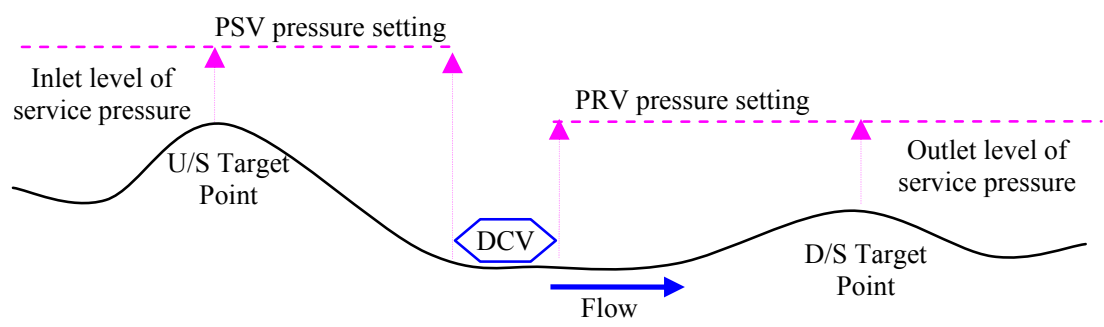

Figure 1: Demand control valve pressure settings.

Therefore when the DCV operates:

- the PSV pilot protects levels of service [6] at the critical node on the upstream side of the valve;

- the PRV pilot maintains levels of service at the critical node on the downstream side of the valve.

The demand control valve maintains a self-regulating system that produces a stable hydraulic profile on both side of the valve for the full diurnal range. A crucial point to make at this point is that the valve is controlled only by the system hydraulics and forms a harmonic element within the network. Automation is achieved solely through the operation of the pilot valves responding to the pressure information in the network and requires no telemetry input.

\subsection{Linear networks}

An autonomous distribution system is controlled via a network of self-optimising nodes (SON). These nodes sense and use the information on the state of their hydraulic environment to modify their mode of operation and regulate the pressure profile [7] across the network (see Figure 2).

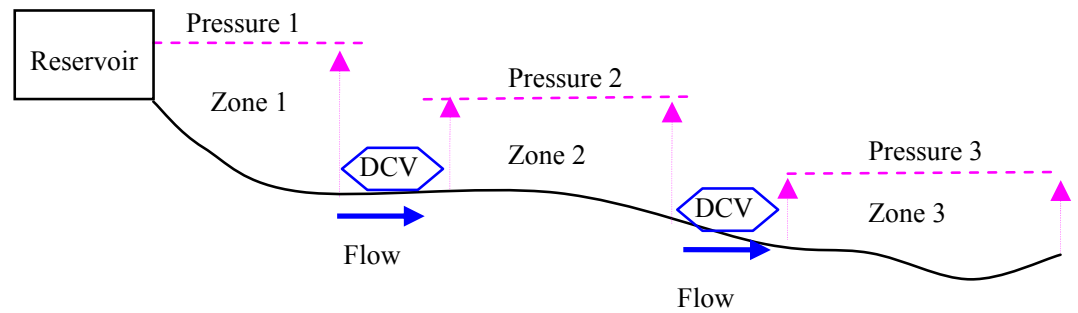

Figure 2: A linear pressure system. 
Each SON consists of a DCV that is hydraulically operated by two pilot regulators. As demand in the system varies the pressure at the SON changes, the valve senses this change and operates to correct the imbalance and maintain a stable hydraulic gradient across the network. By interconnecting these valves, a regulated linear pressure profile is obtained.

This system behaves as follows:

- The first DCV modulates the flow of water from zone 1 to zone 2 whilst protecting and maintaining the pressure in the critical nodes in both zones.

- The second DCV modulates the flow of water from zone 2 to zone 3 whilst protecting and maintaining the pressure in the critical nodes in both zones.

The pressure in all 3 zones is balanced throughout by the interaction of the PSV /PRV pilots on the DCVs.

This process modulates the flow of water through the system which then selfoptimises the pressure in each zone to ensure that a smooth hydraulic gradient is maintained across the whole distribution network throughout the diurnal demand period.

Again this degree of automation is achieved solely through the operation of the pilot regulators alone. The valves are not responding to telemetry signals but to the pressure variation within the network. The information on the system is transmitted hydraulically through the network so no complex algorithms, telemetry or remote control is needed to operate the system.

\subsection{Cross boundary network sustaining valves (NSV)}

A network sustaining valve (NSV) connects two independent supply systems. When the pressure in the one of the systems falls below a minimum value the NSV operates to supply water into this system.

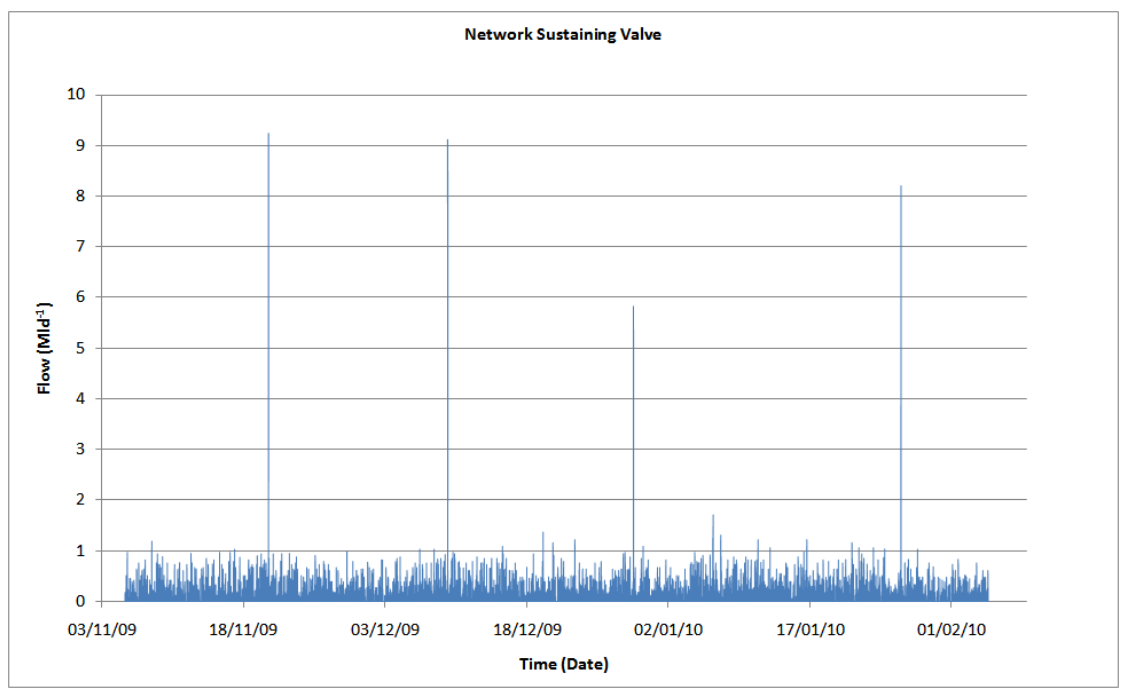

Figure 3: Flow rate through the network sustaining valve. 
Figure 3 shows how, under normal conditions, a flow of approximately 1 Mld is maintained through the valve, this continuous flow maintains water quality in the main.

Over the period shown, the valve has operated in its full PSV mode four times supplying between 6 Mld and 9 Mld to back up the lower pressure system.

This valve is active at all times and as can be seen, is capable of supplying large quantities of water at intermittent periods while still retaining the ability to maintain a low flow for water quality purposes. In addition to the elements above, the valve can also be controlled remotely to allow for network reconfiguration.

\subsection{Reservoir flow control valves (RFCV)}

The flow of water from a reservoir can be limited to the highest peak demand recorded. If a burst occurs on the outlet trunk mains of the RFCV restricts the supply capacity of the reservoir and prevents reservoir drawdown and any air locking of the trunk main.

\subsection{Self optimising network}

The operational advantages obtained from utilising the demand control valve, the network sustaining valve and the reservoir flow control valve are now combined to form an integrated self-optimising network.

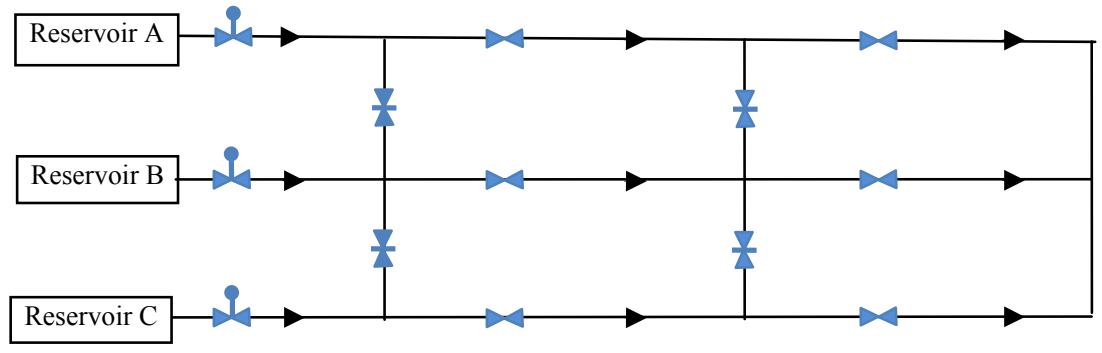

Figure 4: Schematic of a simplified self-optimising network.

The schematic shown in Figure 4 depicts three linear systems A, B and C, with water flowing from left to right. Each linear system uses the same control properties as those described above but is additionally augmented by network sustaining valves (NSV) situated at cross connections to provide a backup from the neighbouring system.

Should a high demand incident occur i.e. a burst or high fire demand the valve on the cross connection will open to supply water from the adjacent system. Again, because of the valve design this can only happen if water is available in the system supplying the water. This will always be the case unless incidents occur in both systems simultaneously. 
The resultant network is now capable of operating autonomously, providing cross system backup and smoothing the hydraulic gradient within the network. Additionally, given the correct control setting, the system will deliver water at a minimum cost throughout the whole network. This aspect of control is discussed in later in this paper.

\subsection{Security of supply: DG levels of service indicators and SIM points}

It would be imprudent to believe that DG level of service infringements will never happen within water distribution system, consequently it would be practical to prepare for these events, contain them and minimise SIM points [8].

To ensure that the least possible disruption occurs within a distribution system, the system must respond automatically to any reduction in network performance and reinforcing areas where incidents occur.

The practice and methodology of such a scheme are detailed below.

\subsubsection{Event containment cells}

The self-optimising network above can be considered as a collection of cells which are interconnected via DCVs. Each valve reacts to the hydraulic parameters upstream and downstream, and regulates the flow of water between the cells.

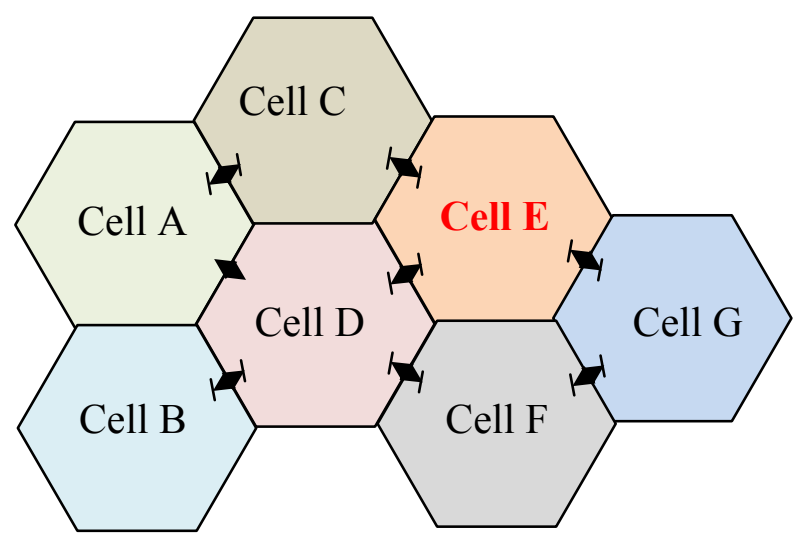

Figure 5: Schematic of a distribution cell structure.

If a burst occurs in any cell it will be supplied from an available adjacent cell. The PSV regulator on the inlet of the DCV will limit the flow of water and protect levels of service in the supplying cell. This ensures that no customers in those cells notice any infringement of DG levels of service associated with, Low pressure (DG2), Supply interruptions (DG3) and restrictions on water use (DG4).

The effects of the burst will be contained within the original cell and only the customers with in this cell will experience levels of service failure. Because 
the pressure in the supplying cells is sustained the number of customer contacts from those cells is eliminated.

The number of level of service failures can be reduced further by installing DCVs to the inlets of the DMAs within each cell. Under these circumstances the level of service failures are contained within the DMA.

With the network reconfigured and the new DCV taking autonomous control of water distribution, hydraulic profiles across the network will become smoothed and consequently the occurrences of levels of service infringements are minimised.

With these measures implemented the security of supply is maintained, DG levels of service infringements are minimised and potential SIM points are not accrued.

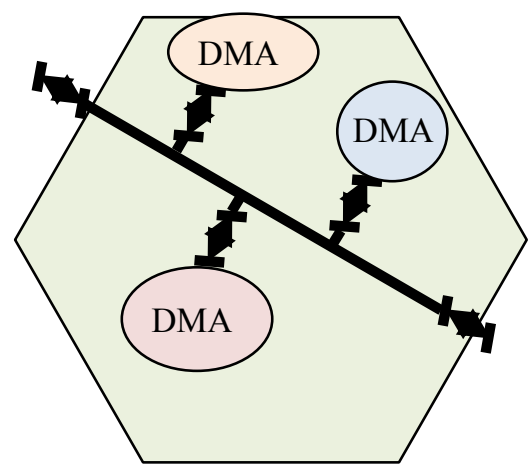

Figure 6: Distribution cell structure.

\subsubsection{Cost minimisation paradigm}

If more than one water source supplies a distribution system then the cost of water delivery can be minimised through the use of controlled pressure management. This process is achieved at the point where the source water enters the distribution system. Here the cheapest water source can be maximised before the more expensive water is used. The mechanics of this process are described below:-

\subsubsection{Cost minimisation physical set up}

The downstream PRV regulators on the DCV controlling the water flow into the distribution system are set to reflect the cost of the water they deliver.

The DCV providing lowest cost water has its PRV set at the nominal pressure for the area.

The DCV providing intermediate cost water has its PRV set at $1 \mathrm{~m}$ less than nominal pressure.

The DCV providing highest cost water has its PRV set at $2 \mathrm{~m}$ less than nominal pressure. 


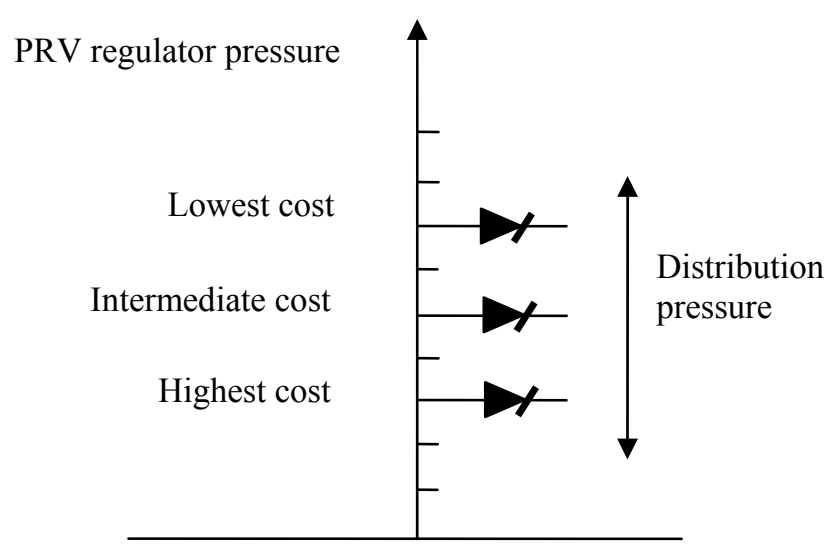

Figure 7: Diagram of PRV setting to cost of water delivery.

\subsubsection{Cost minimisation operational set up}

The PSV setting on the upstream regulator of the first DCV limits the source to its maximum ability to supply whilst maintaining upstream pressure. If demand continues to rise, the pressure on the downstream side of the valve will fall and the second DCV will open and supply the demand area with the intermediate cost water. The supply from the second valve is again limited to the capacity of the upstream source and once this is maximised the final DCV will open supplying the area with the highest cost water. Under this arrangement, water is supplied to the distribution system at a minimum cost.

This mode of operation is not fixed and can be changed at any time, by remotely adjusting the PRV regulator settings. This may be done to alter the water quality parameters of the demand area or because of operational difficulties in any of the supply areas.

\subsubsection{Cost minimisation example}

The cost optimisation model described above has been put into practice and is operating at Nothumbrian Water. At the Mayfair control station in Sunderland City Centre the distribution system is supplied with water from three different sources each of which have different costs. Figure 8 shows the interaction of the valves at St Marks, Otto Terrace, and Mayfair.

The hydraulic interplay between valves is clearly seen with St Marks supplying the bulk of the demand. Otto Terrace supplies water when the flow through St Marks falls and Mayfair picks up the demand that cannot be supplied by the other valves.

Cost minimisation occurs because the lowest cost water is supplied through $\mathrm{St}$ Marks which is dominant. As St Marks valve reaches it maximum capacity to supply water Otto Terrace valve opens and supplies the intermediate cost water. As demand continues to rise, it is supported with the highest cost Mayfair water. 


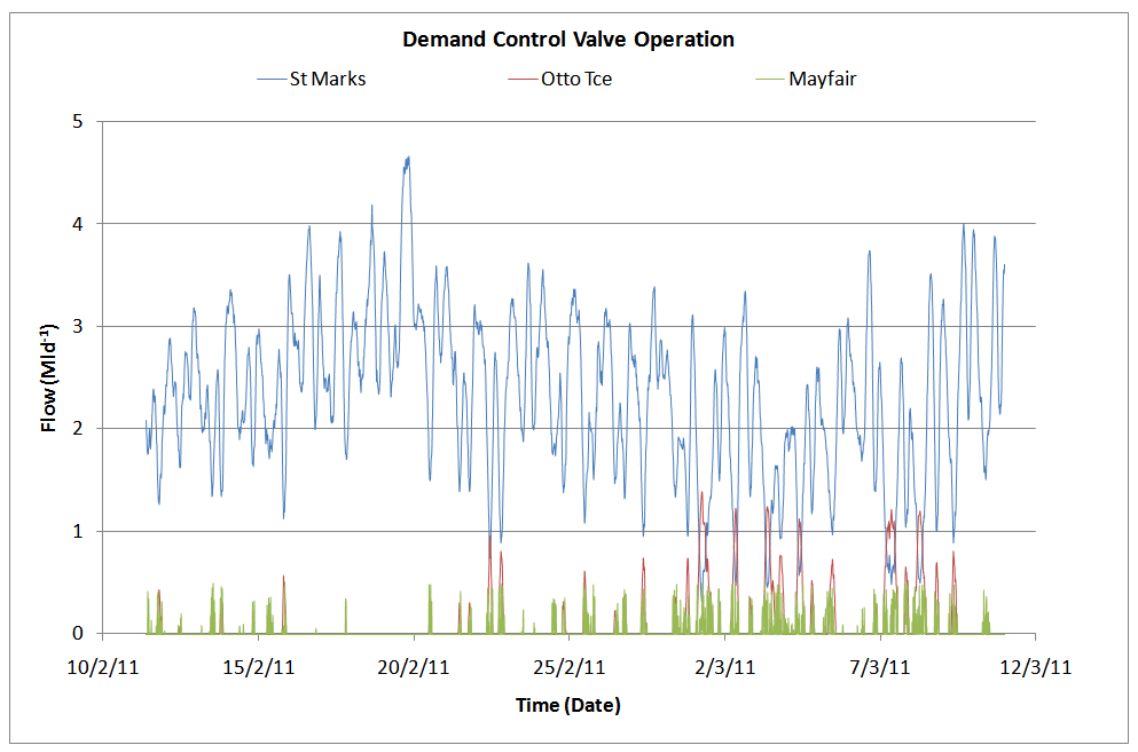

Figure 8: Flow rate through the demand control valves at Mayfair.

If at any time demand on the upstream side of St Marks decreases, additional water automatically becomes available to supply the distribution system. At this point Mayfair and then if required Otto Terrace will throttle their supply volume to a value equal to the additional water being supplied by St Marks.

As demand in the distribution system falls the valves will begin to close in order of their supply costs i.e. Mayfair, Otto Terrace and St Marks. This self compensating method of water supply minimises cost and regulates the downstream pressure to within a two metre band.

\subsection{Cost savings}

The cost savings produced by the self-optimising network in Sunderland city centre are shown in Table 1 below (this data is taken from Figure 8 shown above).

Table 1: Cost savings achieved at Mayfair control.

\begin{tabular}{c|c|c|c}
\hline \multicolumn{1}{c}{ Valve } & Mean flow & Cost & Yearly saving \\
\hline St Marks & $2.3 \mathrm{Mld}$ & $£ 20 / \mathrm{Ml}$ & $£ 58,087$ \\
Otto Tce. & $0.15 \mathrm{Mld}$ & $£ 50 / \mathrm{Ml}$ & $£ 2,219$ \\
Mayfair & $0.63 \mathrm{Mld}$ & $£ 90 / \mathrm{Ml}$ & \\
\hline
\end{tabular}

The first two column of the table show the mean flow and cost of the water supplied through the respective valves. The third column details the yearly savings produced by replacing the most expensive Mayfair water with St Marks water $£ 58,087(\$ 90,644)$ and Otto Terrace water $£ 2,219(\$ 3,462)$. 


\section{Conclusion}

By adopting an integrated approach to developing a company's water distribution system a self-optimising network is created. The system is developed through an exaptation of the existing trunk main system whereby existing principle control valves will be replaced at the end of their asset life with intelligent valves that interact in synchronization with each other and the surrounding hydraulic environment. These valves will be aware of their local and remote environment and will work in unison regulate to maintain a stable hydraulic profile.

All of the elements outlined above, namely demand control valves, cross boundary system valves, linear system control, and self optimising networks, can now all be incorporated into the existing infrastructure to produce an integrated water distribution system. Such a system will produce a hydraulic network with a regulated hydraulic profile and low burst and leakage rates. Additionally the system will automatically self-regulate to minimise the cost of water distribution.

The system is autonomous and will operate independently of external control. Telemetry will be used to monitor the system but not to operate it. Consequently the level of telemetry required can be reduced.

The key issue in developing this strategy is that when a valve specification is made it should not done in isolation but rather in relation to its immediate environment and the operational requirements of the distribution system as a whole.

\section{References}

[1] Prashant Palkar, Shrinivas Patil, Pooja Belagali, Ashish Chougule. Automation in drinking water supply distributed system and testing of water IOSR. Journal of Electronics \& Communication Engineering (IOSR-JECE) ISSN: 2278-2834, ISBN: 2278-8735, pp. 36-38 http://iosrjournals.org/iosrjece/papers/sicete-volume1/8.pdf

[2] Lambert. A. 2000 http://www.geocities.ws/kikory2004/2_Lambert.pdf

[3] CIWEM Water Distribution Network Leakage in the UK http://www.ciwem.org/knowledge-networks/panels/water-supply--quality /water -distribution-network-leakage-in-the-uk.aspx

[4] The Hydraulic Handbook Trevor M. Hunt Elsevier, 1 Jan 1996 - Science p. 157

[5] Flow control valves http://en.wikipedia.org/wiki/Flow_control_valve

[6] OFWAT Levels of Levels of service for the water industry in England and Wales http://www.ofwat.gov.uk/regulating/aboutconsumers/levelsofservice

[7] Nir Naveh, Mike Wiltshire, Pedro Luis Sánchez Rodríguez. Pressure management in water supply networks. 2008. http://www.zaragoza.es /contenidos/medioambiente/cajaAzul/30S7-P3-Nir\%20NavehACC.pdf

[8] OFWAT Service incentive mechanism http://www.ofwat.gov.uk/regulating /aboutconsumers/sim 